\title{
Selection of electrode tips for the resistance spot welding of dissimilar stainless steels
}

\author{
Teerawut Khuenkaew and Kannachai Kanlayasiri * \\ Industrial Engineering Department, Faculty of Engineering, King Mongkut's Institute of Technology Ladkrabang, Bangkok, Thailand
}

\begin{abstract}
This research sought to resolve the issue of electrode tip selection used in the resistance spot welding for two different stainless steel grades under otherwise identical welding parameters: SUS316 and SUS425. The study used a full fractional design in order to perform the analysis of the variables involved, while the quality of the weld was assessed by considering the indentation depth, penetration, and nugget diameter. The results of the study described the nugget diameter and penetration for all welds with the various different electrode tips which met the specified requirements of the customers. A single type of upper R30 and lower R30 electrodes achieved acceptable indentation depth. Ideally, good weld quality would demand a large nugget diameter, shallow indentation, and good penetration, all contribution to an acceptable overall appearance. Both R30 electrode tips are able to achieve the desired quality requirements in the spot welding process.
\end{abstract}

\section{Introduction}

Within the automotive and manufacturing sectors, Resistance spot welding (RSW) is a common approach when joining sheet metal. The creation of joint exhaust pipe parts has made significant use of both ferritic and austenitic stainless steel grades because they have an attractive appearance, can be readily welded, and resist corrosion [1]. When RSW techniques are applied to the dissimilar stainless steel grades 316 and 425, problems can arise when the nuggets are formed because the high temperatures in the molten metal along with high current density distribution can lead to cracking [2]. The outcome can lead to problems for the vehicle parts in the form of excessive noise and vibration, as well as compromised strength. This particular spot welding problem has its influence upon the nugget formation in RSW. In addition, the geometric qualities of the electrode tip are also able to influence the way in which weld nugget formation occurs. This also has a critical effect upon current conduction [3].

The electrode geometry has already been studied for resistance spot welding parameters in terms of nugget growth. Y. Li et al. presented the effect of the cone angle of truncated electrodes in terms of heat and mass transfer in RSW. The result recommended electrode cone angles of $30^{\circ}$ and $45^{\circ}$ in vehicle body production [2]. W. Zhang et al. reported that a planar circular tip diameter of 10 $\mathrm{mm}$ and spherical tip diameter of $70 \mathrm{~mm}$ were the optimal tip electrodes morphology for dissimilar RSW of galvanised high strength steel and aluminium alloy, respectively [4]. Y. Luo et al. studied the calculation of dynamic resistance of nugget growing in RSW. The nugget growth in RSW can be divided into three stages, which were the initial stage, growing stage, and stable stage [5]. T. Khuenkaew and K. Kanlayasiri investigated the use of the Box-Behnken experiment design method with 6 factors for quality characteristics, which are penetration, nugget diameter and nugget area, on the RSW of dissimilar stainless steel grade 316 and grade 425 [6].

The objective of this paper was to determine the electrode tip for weldment quality characteristics, which are penetration $(\mathrm{t} 1, \mathrm{t} 2)$, indentation depth $(\mathrm{h} 1, \mathrm{~h} 2)$ and nugget diameter (di) on the RSW of dissimilar stainless steel grade 316 and grade 425 . The variables used in this study relating to the upper electrode tip shape (UT) and lower electrode tip shape (LT) were flatness and radius, which consider the appearance and macrostructure of weld nuggets in the spot welding process. The full factorial design method and ANOVA were used to investigate the RSW parameter factors on the nugget geometry of RSW.

\section{Materials and Methodologies}

The base materials used were austenitic stainless steel 316 (SUS316) and ferritic stainless steel 425 (SUS425) sheets. Both materials were divided into plates of $45 \times$ $45 \times 1.5 \mathrm{~mm}$. Spot welding was performed using a 50 $\mathrm{kVA}$ AC device, $50 \mathrm{~Hz}$ RSW machine. The spot welding parameters were applied for experimentation as shown in Table 1. The $13 \mathrm{~mm}$ outer diameter of the electrode tips made from $\mathrm{CrCuZr}$ alloy was applied. The spots were taken for appearance analysis. The characteristics of the weld nuggets were measured for all samples of metallographic cross-sections of the weld zone, as shown in Fig. 1 (a). The metallographic samples were ground

* Corresponding author: kannachai.ka@kmitl.ac.th 
and polished, and were then electrolytically etched by $30 \%$ nitric acid solution at $6 \mathrm{~V}$ for $10-15 \mathrm{~s}$ at room temperature. The macrostructures of all samples were assessed using an optical microscope (OM), as shown in Fig. 1 (b). The full factional experiment design was used as the experiment technique (27 runs).

Table 1. Resistance spot welding parameters used in the study.

\begin{tabular}{|c|c|}
\hline Spot welding parameters & Value \\
\hline Electrode force $(\mathrm{kN})$ & 3.0 \\
\hline Welding time (cycle) & 30 \\
\hline Welding current (amperes) & 8,800 \\
\hline
\end{tabular}

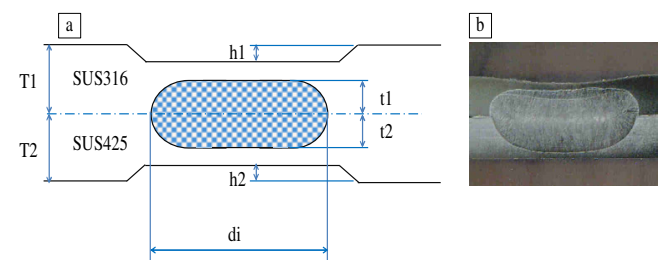

Fig. 1. (a) Schematic illustration of the weld zone

(b) Cross-sectional macrostructure of the weld joint from the electrode UT R30, LT flat

\section{Results and Discussion}

\subsection{The appearance of the weldment}

The appearance of the weldment of SUS316 and SUS425 using the flat type on the upper electrode and a different electrode tip type on the lower electrode is shown. Fig. 2 (a) and Fig. 3 (a) present the appearance with similar results; the results appeared to splash at the edge around the periphery of the contact surface. Fig. 2 (b) and (c) show that the splash of the weld joint appeared in a circular shape. Obviously for each examination, the indentation depth has not occurred. In Fig. 3 (b) and (c) the indentation depth is shown. The indentation depth of LT R16 is higher than LT R30. It can be interpreted that the indentation depth increases with a smaller contact area and higher cone angle of the electrode tip.
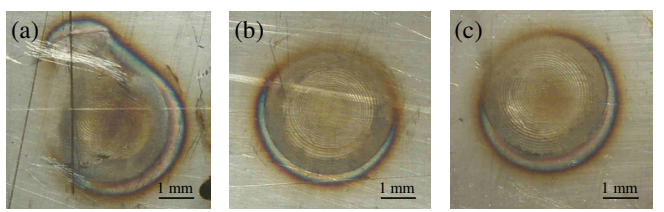

Fig. 2 Appearances of the welded joints of SUS316 and SUS425 by all of UT flat and LT different electrode shapes on SUS316 side (a) UT flat - LT flat, (b) UT flat - LT R 16, (c) UT flat - LT R 30
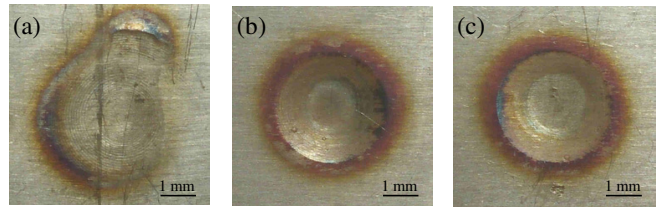

Fig. 3 Appearances of the welded joints of SUS316 and SUS425 by all of UT flat and LT different electrode shapes on SUS425 side (a) UT flat - LT flat, (b) UT flat - LT R 16, (c) UT flat - LT R 30

The appearance of the weldment of SUS316 and SUS425 using R16 type on the upper electrode and different electrode tip types on the lower electrode is shown. Fig. 4 (a-c) shows the appearance of severe expulsion on the SUS316 surface of the welded joint which occurred during welding. This is because of the high current density in a small contact surface, which may generate excessive heat in the small area. The expulsion reaches to the formation of diffused indentation depth. Fig. 5 (a-c) shows the value of indentation depth for LT R16 is the highest, with LT R30, flat, respectively. The expulsion was shown only for the LT R16 type, and clearly UT R16 and LT R16 induced expulsion.
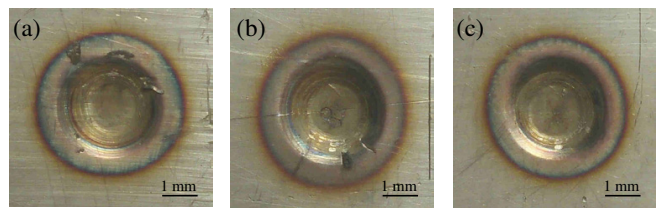

Fig. 4 Appearances of the welded joints of SUS316 and SUS425 by all of UT R16 and LT different electrode shapes on SUS316 side (a) UT R16 - LT flat, (b) UT R16 - LT R 16, (c) UT R16 - LT R 30
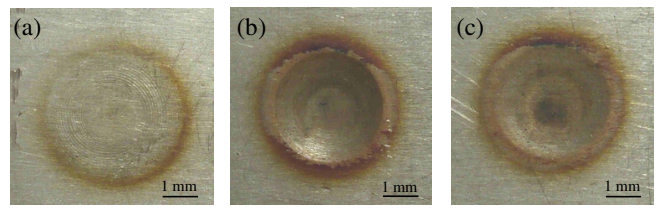

Fig. 5 Appearances of the welded joints of SUS316 and SUS425 by all of UT R16 and LT different electrode shapes on SUS425 side (a) UT R16 - LT flat, (b) UT R16 - LT R 16, (c) UT R16 - LT R 30

The appearance of the weldment of SUS316 and SUS425 using the R30 type on the upper electrode and different electrode tip types on the lower electrode is shown. Fig. 6 (a-c) and Fig. 7 (a-c) clearly show the appearance of the weld nugget. When the electrode tips at each side of the joint are the same, the force delivered is symmetrical, as is the distribution of the current density. This allows the weld nuggets to develop symmetrically within the welded sheets, as Fig 6 (c) and Fig. 7 (c) show. However, during the heating and cooling cycles the heat distribution is asymmetrical. 

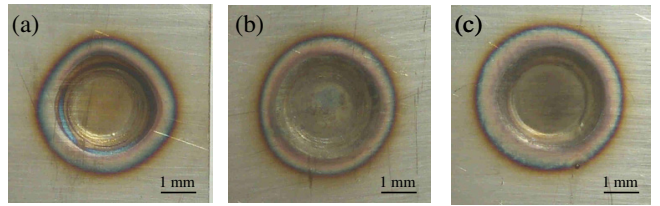

Fig. 6 Appearances of the welded joints of SUS316 and SUS425 by all of UT R30 and LT different electrode shapes on SUS316 side (a) UT R30 - LT flat, (b) UT R30 - LT R 16, (c) UT R30 - LT R 30
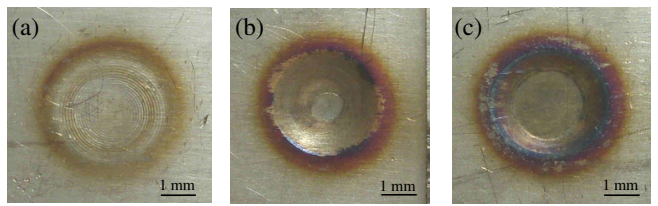

Fig. 7 Appearances of the welded joints of SUS316 and SUS425 by all of UT R30 and LT different electrode shapes on SUS425 side (a) UT R30 - LT flat, (b) UT R30 - LT R 16, (c) UT R30 - LT R 30

In this study, good symmetry was developed in the penetration when using the same electrode tips for each side of the joint, as shown in Fig. 8: R16 (UL R16 - LT R16) and R30 (UL R30 - LT R30).
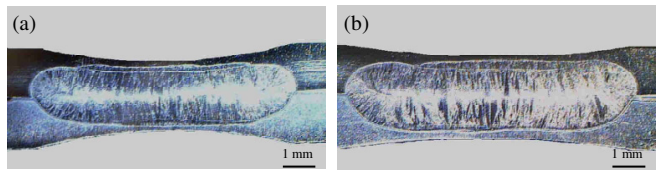

Fig. 8. Cross-sectional macrostructure of the weld joint from symmetry of the electrode tip geometry (a) UT R16 - UT R16, (b) UT R30 - LT R 30

Comparisons of the appearances were made for the two different types. When the same R30 electrode tip is used on both sides, the overall appearance is improved due to good indentation depth, increased nugget diameter, and lower expulsion. The parts for vehicle exhaust pipes are predominantly welded on their curved surfaces, and therefore the radius type of electrode geometry would be the best choice for spot welding in these circumstances.

\subsection{Weldment quality characteristics}

MINITAB statistical software was used to create and analyse the experiment data. The significance for the welding quality ( $t 1, \mathrm{t} 2, \mathrm{~h} 1, \mathrm{~h} 2$, di) was evaluated using the $p$-value. In this case the p-value was smaller than the confidence level of 0.05 , indicating the statistical significance of the model. The $\mathrm{R}^{2}$ values of this analysis were $99.83 \%, 99.81 \%, 99.00 \%, 97.19 \%$ and $99.83 \%$, respectively, while the adjusted $\mathrm{R}^{2}$ values of the model were $99.76 \%, 99.72 \%, 98.55 \%, 95.94 \%$ and $99.75 \%$, respectively. These values were relatively moderate to high.

\subsubsection{Penetration}

The effects of different electrode tips on the penetration of the spot welded joints were illustrated in Fig. 9 (a) and (b). Penetration should be kept greater than $20 \%(>0.3$ $\mathrm{mm}$ ) of the thinnest thickness of the material. Fig. 9 (a) shows the penetration 1 . The penetration 1 of all results was higher than $0.3 \mathrm{~mm}$. The maximum and minimum values of penetration 1 used UT R30 - LT flat (1.18 mm), UT R16 - LT R30 (0.66 mm), respectively. Fig. 9 (b) shows the penetration 2. The maximum and minimum values of penetration 2 used UT R16 - LT flat $(1.26 \mathrm{~mm})$, UT flat - LT R16 $(0.75 \mathrm{~mm})$, respectively. The penetration of the SUS425 side was higher than that of the SUS316 side because of the difference in the mechanical and physical properties.

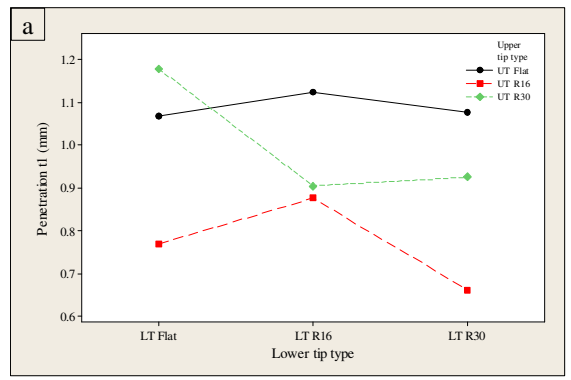

Fig. 9a. Interaction plot for penetration $\mathrm{t} 1$

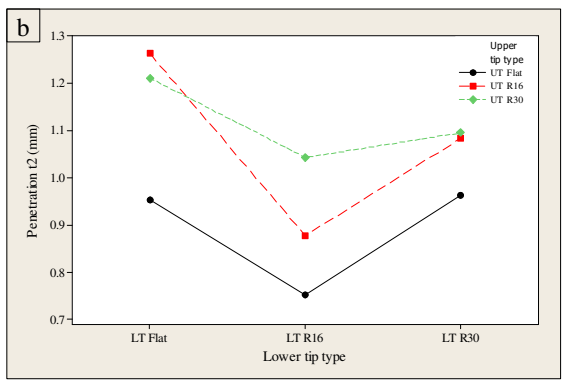

Fig. 9b. Interaction plot for penetration $\mathrm{t} 2$

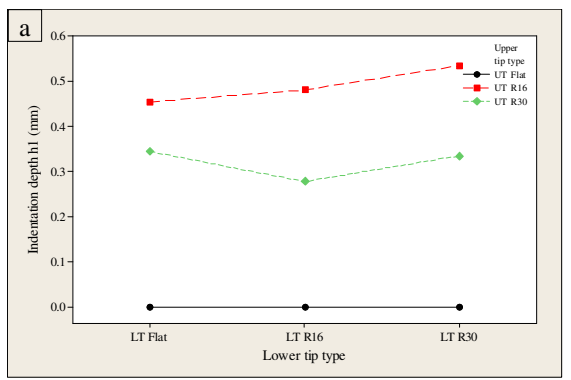

Fig. 10a. Interaction plot for indentation depth $\mathrm{h} 1$

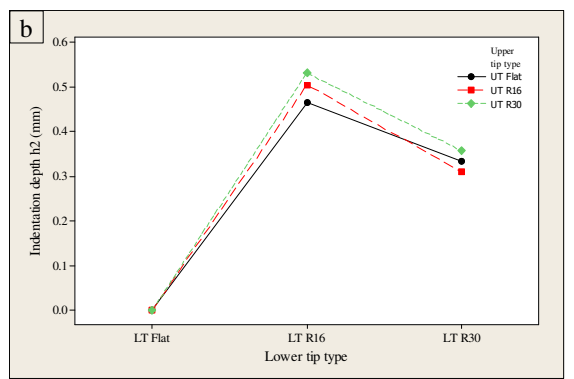

Fig. 10b. Interaction plot for indentation depth $h 2$ 


\subsubsection{Indentation depth}

In the general designs, the indentation depth was less than $30 \%(<0.45 \mathrm{~mm})$ of the thickness of the sheet. Fig. 10 (a) and (b) shows the indentation depth of the spot welded joints. For both the UT and LT of flat electrode tips there was no indentation depth. The electrode flat shape was used to improve the weld surface quality, conversely the indentation depth of UT R16 and LT R30 electrode tips reached the maximum value $(0.53 \mathrm{~mm})$ in all experiments because the cone electrode R16 offers a higher angle and smaller contact surface, which could not improve the cooling ability of the electrode. Obviously for each examination, the expulsion occurs. The indentation depth of UT 30 and LT R30 achieved the target $(0.33 \mathrm{~mm})$.

\subsubsection{Nugget diameter}

The nugget diameter was larger than $4 \sqrt{ } \mathrm{t}(>5.0 \mathrm{~mm})$, in which $t$ represents the thinnest part of the thickness of the sheet. Fig. 11 shows the nugget diameter of the spot welded joints.

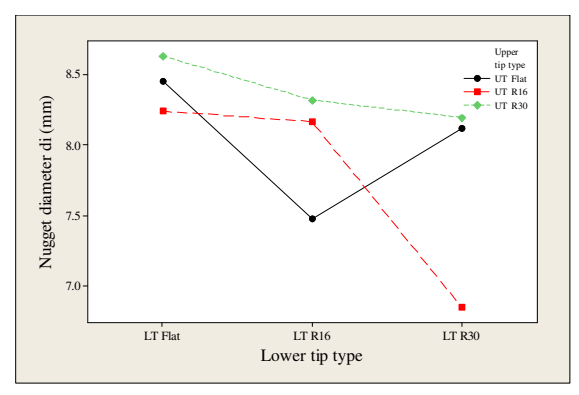

Fig. 11. Interaction plot for nugget diameter

The nugget diameter of all results was larger than 5.0 $\mathrm{mm}$. The maximum and minimum values of nugget diameter used UT R30 - LT flat $(8.63 \mathrm{~mm})$, UT R16 LT R30 (6.85 mm), respectively. The customer requirements demanded good weldment quality through criteria such as good penetration, a large nugget diameter, shallow indentation depth, lower expulsion, adequate strength and a good overall weld appearance. The RSW process used electrode tips of differing geometry, while the welding involved the same materials with the same thickness, under identical welding parameters. Therefore, the main influence upon differences in nugget formation would be electrode geometry. The current density determines the contact surface between the workpiece and the electrodes, and the use of flat type electrodes to increase the contact surface can serve to increase the nugget shape. Concentric electrodes present one concern because of a lack of homogeneity in the distribution of the current density when flat type electrodes are used. This results in the greatest current densities being found at the edges of the contact surface. The outcome is that when weld nuggets are formed using a larger contact surface electrode on the face side, whether UT flat or R30, the nugget edges will protrude from the electrode at the smaller contact surface [7]. Expulsion occurs when the upper or lower electrode is of the R16 type, because of the increased current density within a small contact area, since this can create excessively high temperatures. The expulsion leads to the creation of diffuse indentation [8].

\section{Conclusions}

In this study, electrode tips used in the RSW process were selected for use with varying stainless steel grades in order to vary the quality of the nuggets formed. Furthermore, the morphology of the electrode has to be chosen on the basis of the task to be performed. In this case, the welding of vehicle exhaust pipe parts must be carried out on a curved surface, and therefore the radius electrode geometry should be selected for the spot welding process. In such circumstances, R30 can be used for both the upper and lower electrode tip geometry in order to achieve suitable weldment quality in the RSW process.

\section{Acknowledgements}

The researchers are grateful to Yokoyama Kogyo Thailand Co., Ltd for providing access to the RSW machine. The authors would also like to express their thanks to the Faculty of Engineering of King Mongkut's Institute of Technology Ladkrabang who offered support in covering the costs incurred in conducting this research.

\section{References}

1. D. Kianersi, A. Mostafaei, A. Ali Amadeh, Mater. Des. 61 251-263 (2014).

2. Y. Li, Z. Wei W, Y. Li, Q. Shen, Z. Lin, Int. J. Heat Mass Tranf. 65 400-408 (2013).

3. D.Min, L.Shi-sheng, H. Hong, P. Tao, Z. Pei-Lei, Mater. Des. 52 353-358 (2013).

4. W. Zhang, D. Sun, L. Han, Y. Li, Mater. Des. 85 461-470 (2015).

5. Y. Luo, R. Wan, Z. Yang, X. Xie, Measuement. 87 18-28 (2016).

6. T. Khuenkaew, K. Kanlayasiri, ICMM, International Conference on Mechatronics and Manufacturing (2018).

7. W. Marzur, A. Kyriakopoulos, N. Bott, D. West, J. Manuf. Process. 22 60-73 (2016).

8. H. Zhang, X. Qiu, Y. Bai, F. Xing, H. Yu, Y. shi, Mater. Des. 63 151-158 (2014). 